\title{
Preparation and Characterization of Sodium Alginate/Chitosan Composite Nanoparticles Loaded with Chondroitin Sulfate
}

\author{
X. B. Yin, Q. Z. Yu (i), B. W. Li, and C. D. Zhang \\ Department of Materials and Textile Engineering, Key Laboratory of Yarn Materials Forming and Composite Processing \\ Technology of Zhejiang Province, Jiaxing University, Jiaxing, Zhejiang 314001, China
}

Correspondence should be addressed to Q. Z. Yu; w2003yqz@126.com

Received 26 November 2020; Revised 27 April 2021; Accepted 26 June 2021; Published 8 July 2021

Academic Editor: Mikhail Zheludkevich

Copyright $\odot 2021$ X. B. Yin et al. This is an open access article distributed under the Creative Commons Attribution License, which permits unrestricted use, distribution, and reproduction in any medium, provided the original work is properly cited.

The chondroitin sulfate (CS)/sodium alginate (SA)/chitosan nanoparticles were prepared by electrostatic spraying technology. Laser particle size analyzer measurements indicated that the size of the particles is strongly affected by the weight ratio of raw materials, receiver rotation speed, injection rate, and so on. The size increased and size distribution moved towards the direction of large particle size with the decrease of applied voltage and increased with the increase of the flow rate and weight ratio of SA to CS; there is an optimal value for the effect of stirring rate on particle size and particle size distribution. The effect of different weight ratios of raw materials and $\mathrm{pH}$ on the sustained release rate of the product was investigated using a UV-visible spectrophotometer. Results showed the cumulative release rate of these particles was weight ratio of SA to CS and pH-dependent. The cumulative release rate of the CS/SA/chitosan composite nanoparticles with a weight ratio of SA to CS of $2: 1$ was higher at pH 6.8 than that at $\mathrm{pH}$ 1.2. By optimizing the process parameters, the CS/SA/chitosan composite particles were prepared, in which most of CS could pass through the stomach and release in the intestinal tract.

\section{Introduction}

Nanoparticles (NPs) will make drugs delivery more convenient in human body [1]. Adding small bioactive molecules to nanoscale transport system may increase the bioavailability and single-cell therapeutic effect of the drug and reduce the clinical dosage. Studies $[2,3]$ have shown that the NPs size determines its extravasation from blood vessels, with the capillary pore size posing as the upper size limit. After extravasation, the smaller NPs are widely dispersed and show deeper penetration in the interstitium relative to larger NPs that are localized in perivascular space. Generally, a size less than $200 \mathrm{~nm}$ is favorable for extravasation and interstitial transport.

In recent years, extensive research has been conducted on the role of chondroitin sulfate (CS) in targeting cancer cells. Apart from the targeting effect on tumor cells via CD44 [4], investigations [5-9] have also shown that CS also has direct anticancer, anti-inflammatory, and immunomodulatory effects and chondroprotective activity and accounts for cell adhesion regulation. These properties have promoted CS as a promising for the development of biomaterial for cartilage regeneration and tissue engineering [10]. However, the excellent hydrophilicity and high hydrodynamic volume in vivo circulation of CS inhibit its interaction with plasma proteins and cells, which limits its application in medicine and pharmaceutical preparations. Moreover, CS cannot self-assemble into nanomaterials in water. It must be hydrophobically modified by hydroxyl and phenol groups in CS for nanomaterials preparation.

Chitosan nanoparticles are widely used for several biomedical applications as a drug delivery system. The positively charged amino group of chitosan nanoparticles, the polyanionic natural polymer, forms complexes with negatively charged polyelectrolytes to spontaneously result in nanoparticle formation $[3,11,12]$. Chitosan is poor solubility in water or organic solvents [13]. So, it is an ideal material used to hydrophobically modify for CS. In contrast, 
sodium alginate (SA) is a naturally derived, nontoxic, watersoluble, and biocompatible polysaccharide, which forms $\mathrm{pH}$ responsive hydrogels in the presence of divalent cations such as $\mathrm{Ca}^{2+}, \mathrm{Mg}^{2+}$, and $\mathrm{Ba}^{2+}$. The interaction between functional groups of alginate and divalent cations results in the formation of crosslinks according to the "egg-box" model. The crosslinked structure of alginate has been used to produce drug delivery systems to release various drugs, proteins and active agents from the matrix $[14,15]$.

In this study, CS was used as an antitumor immunopotentiator. In order to further improve the efficacy of CS as an antitumor immunopotentiator and ensure CS to pass through the stomach and be released in the intestinal tract, the SA was used to coat the CS by dissolving them in deionized water to form hydrogels firstly. Then, chitosan was coated outside the hydrogels through electrostatic attraction to form CS/SA/chitosan composite nanoparticles. In order to obtain the composite nanoparticles with a size of less than $200 \mathrm{~nm}$, the influences of the content of components and the process parameters on the size and the swelling behavior of the composite micro/nanoparticles, and the release rate of CS of carrier materials were studied systematically.

\section{Materials and Methods}

2.1. Materials and Reagents. Chitosan and SA were purchased from MACKLIN (chitosan, high viscosity > $400 \mathrm{MPa}$.s; sodium alginate, biochemical grade, used to fix cells, enzymes, etc.) and used as received. CS was purchased from SigmaAldrich (USA). The other reagents and solvents were of analytical grade.

\subsection{Preparation of SA/Chitosan Composite Micro/Nano-} particles Containing CS. SA/chitosan composite micro/ nanoparticles containing CS were prepared as follows. The weight ratio of SA to chitosan was $2.56: 1$, and the weight ratio of SA to CS was varied from $5: 1,2: 1$, to $1: 1.23 .34 \mathrm{mg}$ chitosan, which was slowly added to $120 \mathrm{~mL}$ deionized water in a beaker. Then, $0.6 \mathrm{~mL}$ glacial acetic acid was added dropwise to the chitosan aqueous dispersion solution after the chitosan was evenly dispersed. Then, $0.2 \mathrm{~g}$ calcium chloride was added to the above solution and stirred at high speed until the calcium chloride was dissolved thoroughly. The obtained solution was the receiving solution. Moreover, $59.6 \mathrm{mg}$ SA was slowly dissolved in $50 \mathrm{~mL}$ deionized water. Then, predetermined CS was added according to the weight ratio of SA to CS of $5: 1,2: 1$, and $1: 1$, respectively. The mixed solution was held in a syringe for electrospraying with the voltage of 13,16 , and $18 \mathrm{kV}$, respectively. A syringe pump was employed to control the flow rate of $0.3,0.6,1.0$, and $1.2 \mathrm{~mL} / \mathrm{h}$, respectively. A beaker filled with receiving solution was used to collect the particles and the collected distance was $6.0 \mathrm{~cm}$. The stirring rate of the receiver was 500 , $600,800,1000$, and $1200 \mathrm{r} / \mathrm{min}$. Experiments were carried out after turning on the ultraviolet lamp for at least $2 \mathrm{~h}$. After spraying, the receiver was taken out and the product was slowly neutralized with sodium bicarbonate firstly. Then, it was poured into the centrifugal tube and centrifuged at
$13,500 \mathrm{rpm}$ at room temperature for $30 \mathrm{~min}$. The supernatant was removed and the nanoparticles sediment (precipitant) was washed by distilled water after centrifugation. This procedure was repeated three times. At last, most of the nanoparticles sediments (precipitants) were freeze-dried and saved below $4^{\circ} \mathrm{C}$. A small amount of the precipitants was dispersed in $5 \mathrm{~mL}$ PBS buffer solution to measure the size and zeta potential of the particles.

In order to facilitate the reader's understanding, we have created a schematic diagram that reflects how the nanoparticles were assembled and how the CS was encapsulated and released. Please see Figure 1 for details.

2.3. Characterization. A Malvern Zetasizer Nano ZS (Zetasizer Nano ZS) instrument that utilizes dynamic light scattering (DLS) and laser doppler velocimetry (LDV) was used to measure the size and zeta potential of the particles, respectively. The morphologies of the composite particles were observed through S-4800 scanning electron microscopy (SEM). FTIR spectroscopy was carried out with a 470 FTIR at transmission mode in the resolution of $4 \mathrm{~cm}^{-1}$.

The swelling behavior of nanoparticles was characterized under the conditions simulating $\mathrm{pH}$, temperature, and time likely to be encountered during transit in the gastrointestinal tract. For this purpose, buffer solutions at a $\mathrm{pH}$ of 1.2 or 6.8 were prepared according to European Pharmacopeia 8.0 [16], where buffer solution at a $\mathrm{pH}$ of 1.2 consisted of $\mathrm{HCl}$ and $\mathrm{NaCl}$ to simulate gastric medium and buffer solution at a $\mathrm{pH}$ of 6.8 consisted of $\mathrm{NaOH}$ and $\mathrm{KH}_{2} \mathrm{PO}_{4}$ to simulate intestinal medium. The concentrations of buffer solutions were $0.1 \mathrm{M}$ and the $\mathrm{pH}$ values of buffer solutions were checked with a $\mathrm{pH}$ meter. The dried nanoparticles were weighed and immersed in $50 \mathrm{~mL}$ of buffer solution at a $\mathrm{pH}$ of 1.2 or 6.8 for $8 \mathrm{~h}$. Swelling experiments were carried out at $37 \pm 0.5^{\circ} \mathrm{C}$. The micro/nanoparticles were removed from the buffer solutions periodically, blotted with a filter paper to remove excess water, and weighed. The swelling ratio (SR) and equilibrium swelling ratio (ESR) values were calculated using the following equations, respectively:

$$
\begin{array}{r}
\mathrm{SR}=\frac{\left(W_{t}-W_{d}\right)}{W_{d}}, \\
\mathrm{ESR}=\frac{\left(W_{s}-W_{d}\right)}{W_{d}},
\end{array}
$$

where $W_{t}$ is the weight of the micro/nanoparticle at time $t$ during swelling, $W_{s}$ is the weight of the swollen micro/ nanoparticle, and $W_{d}$ is the weight of the dried micro/ nanoparticle.

The release of CS from the prepared CS/SA/chitosan composite micro/nanoparticles was studied at the same conditions used in the swelling studies. $5 \mathrm{mg} \mathrm{SA} /$ chitosan composite micro/nanoparticles containing CS were dissolved in $5 \mathrm{~mL}$ PBS buffer solution. The above solution was poured into a dialysis bag. The dialysis bag was immersed in a beaker with $50 \mathrm{~mL}$ shaking PBS buffer solution at a $\mathrm{pH}$ of 1.2 and 6.8, respectively, and was allowed to be shaken at a rate of $100 \mathrm{rpm}$. At predetermined time points $(1,2,3,4,5,6$, 


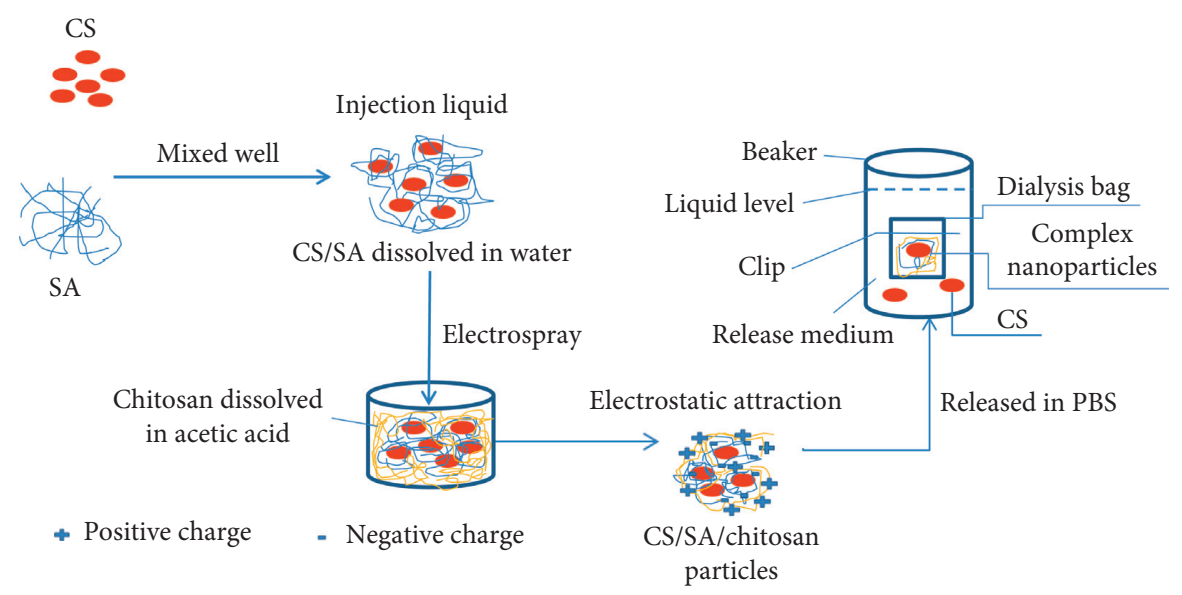

FIgURE 1: The schematic diagram of nanoparticle assembly and encapsulation and release of CS.

$7,8,9,10,11$ and $12 \mathrm{~h}$ ) after starting incubation, samples of $5 \mathrm{~mL}$ from the released medium were taken out and equal volume of fresh buffered solution at $37 \pm 0.5^{\circ} \mathrm{C}$ was added to the release medium to maintain sink conditions. The amount of CS released from composite micro/nanoparticles was measured spectrophotometrically at $220 \mathrm{~nm}$. The percent cumulative release was calculated according to the following:

$$
\text { Cumulative release } \%=\left(\frac{M_{t}}{M_{\infty}}\right) \times 100 \text {, }
$$

where $M_{t}$ is the amount of CS released from composite micro/nanoparticles at time $t$ and $M_{\infty}$ is the total amount of CS loaded in the micro/nanoparticles.

2.4. Statistical Analysis. All experimental data were expressed as the mean \pm standard deviation of at least three independent experiments.

\section{Results and Discussion}

\subsection{Morphology Observation and the Size Distribution in Water}

3.1.1. The Effect of Flow Rate. Fixing the voltage of $16 \mathrm{kV}$, the stirring rate of $800 \mathrm{rpm}$, the collected distance of $6 \mathrm{~cm}$, and the weight ratio of SA to CS of $5: 1$, Figures 2(a)-2(d) show the surface morphology of SA/chitosan micro/nanoparticles loaded with CS at different flow rates. The shape and size of the particle were changed with the variation of flow rate. When the flow rate was lower than $0.6 \mathrm{~mL} / \mathrm{h}$, the shape of obtained SA/chitosan nanoparticles loaded with CS was a sphere with a diameter of about from $20 \mathrm{~nm}$ to $497 \mathrm{~nm}$, as shown in Figures 2(a) and 2(b). While the flow rate was $1.0 \mathrm{~mL} / \mathrm{h}$ (Figure 2(c)), the obtained micro/nanoparticles revealed multiple shapes (special-shaped microspheres), such as cylindrical particles with a diameter of about $50 \mathrm{~nm}$ and height of about $200 \mathrm{~nm}$, isosceles triangle, and equilateral triangle, revealing their irregular shape and well distributed and nonadherent nature. As the flow rate was $1.2 \mathrm{~mL} / \mathrm{h}$ (Figure 1), most of the obtained micro/ nanoparticles were cylindrical with a diameter of about $60 \mathrm{~nm}$ and height of about $100 \mathrm{~nm}$.

The average size and size distribution of the obtained particles were shown in Figures 3(a) and 3(b). As the flow rate increased from $0.3 \mathrm{~mL} / \mathrm{h}$ to $1.2 \mathrm{~mL} / \mathrm{h}$, the average size of the particles increased from about $90 \mathrm{~nm}$ to $600 \mathrm{~nm}$ with the increase of the flow rate, indicating that the slower the flow rate was, the smaller the average size of particles was. The average size of the particles increased with the increase of the flow rate, although the increase in rate of size obtained below $0.6 \mathrm{~mL} / \mathrm{h}$ was slower than that obtained above $0.6 \mathrm{~mL} / \mathrm{h}$. These results indicate that the shape and size of SA/chitosan nanoparticles loaded with CS were strongly affected by the flow rate of injection fluid. The reason may be that the CS/SA droplets are subject to surface tension and fluid resistance of chitosan hydrogel when they collide with chitosan. Under certain voltage and collect distance, the size and weight of CS/SA droplets increased with the increase of the flow rate, resulting in the different surface tension and fluid resistance of chitosan hydrogel film to which CS/SA droplets were subjected.

3.1.2. The Effect of Stirring Rate. Fixing the voltage of $16 \mathrm{kV}$, the flow rate of $0.6 \mathrm{~mL} / \mathrm{h}$, the collected distance of $6 \mathrm{~cm}$, and the weight ratio of SA to CS of $5: 1$, the stirring rate was varied from $500 \mathrm{rpm}, 600 \mathrm{rpm}, 800 \mathrm{rpm}$, to $1200 \mathrm{rpm}$, respectively. The surface morphology of SA/chitosan micro/ nanoparticles loaded with CS at different stirring rates is shown in Figures 2(b), 2(e), and 2(f). It can be found that the shape was also changed with the variation of stirring rate. When the stirring rate was lower than $800 \mathrm{rpm}$, most of the shapes of obtained SA/chitosan nanoparticles loaded with CS were spheres with a diameter of about from $153 \mathrm{~nm}$ to $413 \mathrm{~nm}$, as shown in Figures 2(b) and 2(e). While the stirring rate was $1000 \mathrm{rpm}$ (Figure 2(f)), the obtained micro/ nanoparticles revealed multiple shapes (special-shaped microspheres), such as sphere particles with a diameter of about $20 \mathrm{~nm}$; right triangle with about $233 \mathrm{~nm}$ and $360 \mathrm{~nm}$ sides; equilateral triangle with a side length of $120 \mathrm{~nm}$, revealing their irregular shape.

The average sizes and size distribution of obtained SA/ chitosan micro/nanoparticles loaded with CS are shown in 

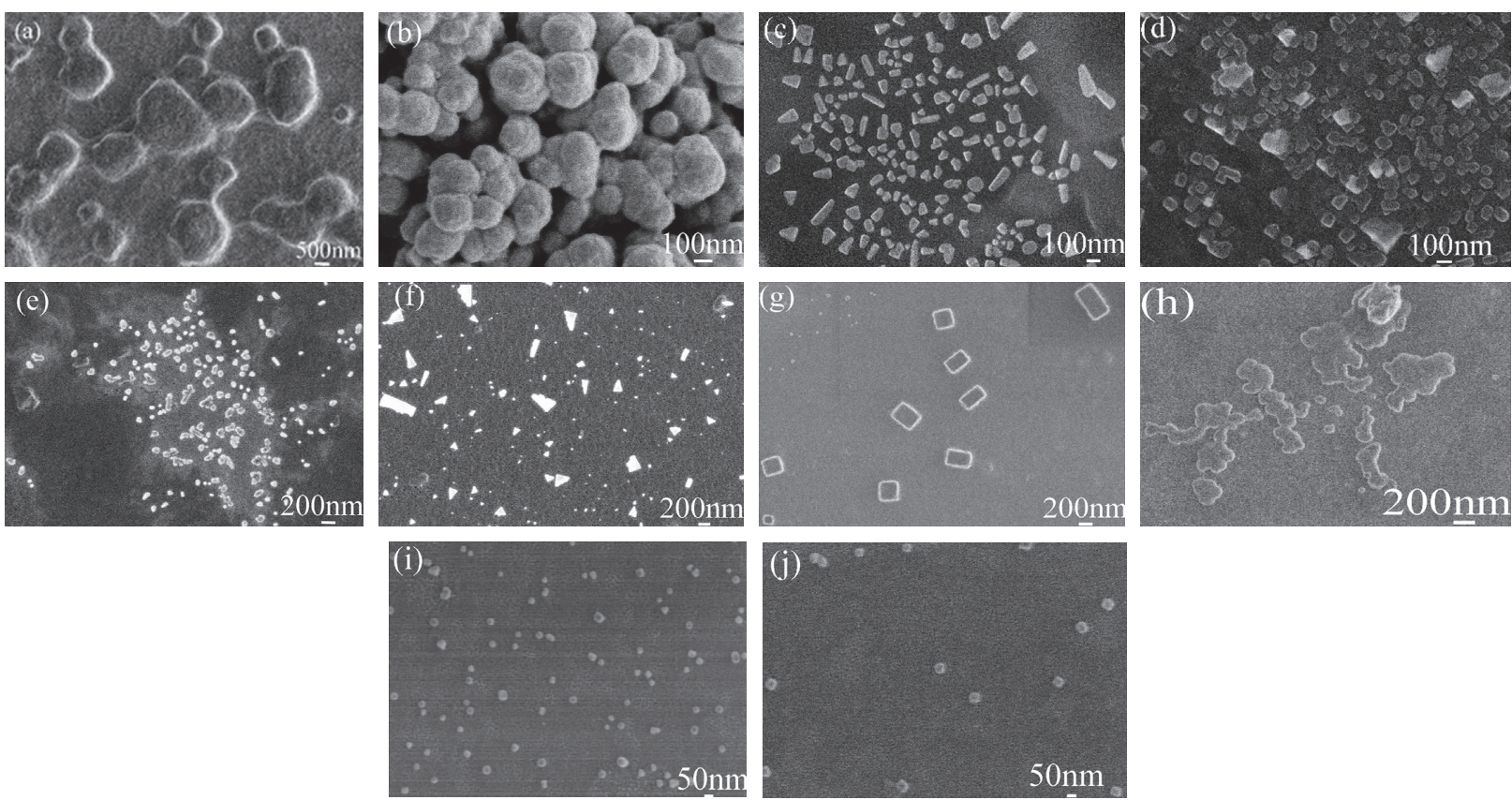

FIGURE 2: SEM images of SA/chitosan micro/nanoparticles loaded with CS prepared by different process parameters. (a)-(d) The voltage of $16 \mathrm{kV}$, the stirring rate of $800 \mathrm{rpm}$, and the weight ratio of SA to CS of $5: 1$ at flow rate of (a) $0.3 \mathrm{~mL} / \mathrm{h}$; (b) $0.6 \mathrm{~mL} / \mathrm{h}$; (c) $1.0 \mathrm{~mL} / \mathrm{h} ;$ (d) $1.2 \mathrm{~mL} /$ h. (e, f) The voltage of $16 \mathrm{kV}$, flow rate of $0.6 \mathrm{~mL} / \mathrm{h}$, and the weight ratio of SA to CS of $5: 1$, with a stirring rate of (e) $600 \mathrm{rpm}$ and (f) $1000 \mathrm{rpm}$. (g, h) The stirring rate of $800 \mathrm{rpm}$, flow rate of $0.6 \mathrm{~mL} / \mathrm{h}$, and the weight ratio of SA to CS of $5: 1$, with applied voltages of (g) $13 \mathrm{kV}$ and (h) $18 \mathrm{kV}$. (i, j) The stirring rate of $800 \mathrm{rpm}$, flow rate of $0.6 \mathrm{~mL} / \mathrm{h}$, applied voltages of $16 \mathrm{kV}$, and the weight ratio of SA to CS of (i) $2: 1$; (j) $1: 1$.

Figures 3(c) and 3(d). Figures 3(c) and 3(d) show that when the stirring rate was between $500 \mathrm{rpm}$ and $800 \mathrm{rpm}$, the average size of the particles decreased from about $753 \mathrm{~nm}$ to $491 \mathrm{~nm}$ with the increase of the stirring rate. While the stirring rate further increased from $800 \mathrm{rpm}$ to $1200 \mathrm{rpm}$, the average size of particles increased from about $491 \mathrm{~nm}$ to $4800 \mathrm{~nm}$, indicating the optimum stirring rate was about $800 \mathrm{~nm}$ to obtain small-sized nanoparticles. It may be because when the stirring rate was lower than the optimum value, the droplets dropped into chitosan and could not disperse and stick together. On the contrary, as the rotational speed was higher than a certain optimum value, the dispersed droplets would have collided together.

3.1.3. The Effect of Applied Voltage. Fixing the collected distance of $6 \mathrm{~cm}$, the weight ratio of SA to CS of $5: 1$, and the stirring rate of $800 \mathrm{rpm}$, when the flow rate was $0.6 \mathrm{~mL} / \mathrm{h}$, the surface morphology of SA/chitosan micro/nanoparticles loaded with CS at different applied voltages is shown in Figures 2(b), 2(g), and 2(h). Figures 2(b), 2(g), and 2(h) show that the shape also changed with the variation of applied voltage. When the applied voltage was $13 \mathrm{kV}$, the shape of obtained SA/chitosan nanoparticles loaded with CS was a rectangle with a length of 400-600 nm and a width of $270 \mathrm{~nm}$ or a square with a side length of $70-300 \mathrm{~nm}$, as shown in Figure 2(g). While the applied voltage was $16 \mathrm{kV}$ (Figure 2(b)), the obtained micro/nanoparticles had a sphere shape with a diameter of about $153 \mathrm{~nm}$ to $413 \mathrm{~nm}$. Moreover, as the applied voltage was $18 \mathrm{kV}$ (Figure 2(h)), sphere particles with a diameter of about $30-50 \mathrm{~nm}$ were formed and a large number of particles gathered together.

The size distributions by $(e)$ intensity and $(f)$ volume at different applied voltages are shown in Figures 3(e) and $3(\mathrm{f})$. It can be seen that the size distribution of particles moved to a smaller direction with the increase of applied voltage. When the applied voltage was increased from $13 \mathrm{kV}$ to $18 \mathrm{kV}$, the average size of the particles was decreased from $760.8 \mathrm{~nm}$ to $51.9 \mathrm{~nm}$, and the size distribution of particles from $514.09-66.9 \mathrm{~nm}$ to $43.3-60.2 \mathrm{~nm}$. It is mainly because droplets will be smaller and smaller with the increase of electric field force resulting from the increase of applied voltage when other parameters are fixed.

3.1.4. The Effect of Weight Ratio of SA to CS. Fixing the collected distance of $6 \mathrm{~cm}$, the stirring rate of $800 \mathrm{rpm}$, the flow rate of $0.6 \mathrm{~mL} / \mathrm{h}$, and the applied voltage of $16 \mathrm{kV}$, the weight ratio of SA to CS varied from $5: 1,2: 1$, to $1: 1$, respectively. The surface morphology of SA/chitosan micro/ nanoparticles loaded with CS at different weight ratios is shown in Figures 2(b), 2(i), and 2(j). Figures 2(b), 2(i), and $2(j)$ showed that the shape of all the particles obtained from different applied voltages was sphere. When the weight ratio of SA to CS was $5: 1$, the diameter of the sphere was about $153-326 \mathrm{~nm}$. Moreover, as the weight ratios of SA to CS were $2: 1$ and $1: 1$, the diameter of the spheres decreased to about $30-60 \mathrm{~nm}$ and $30-50 \mathrm{~nm}$, respectively. 


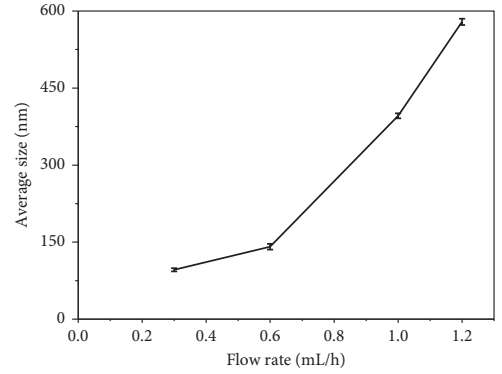

(a)

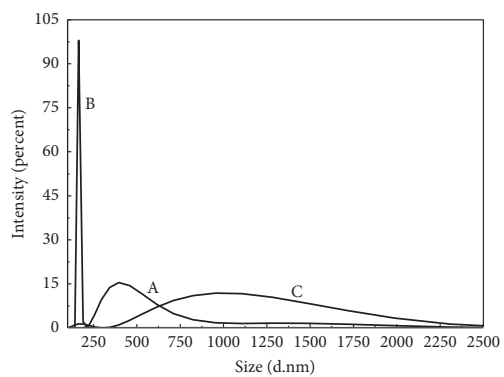

(d)

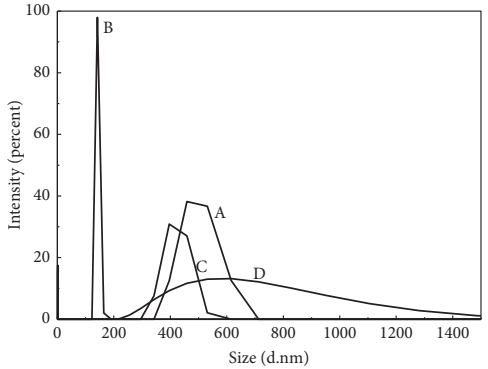

(b)

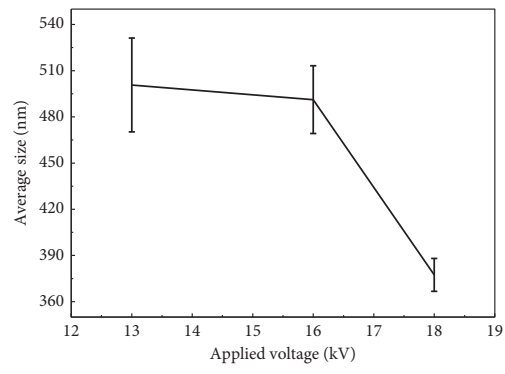

(e)

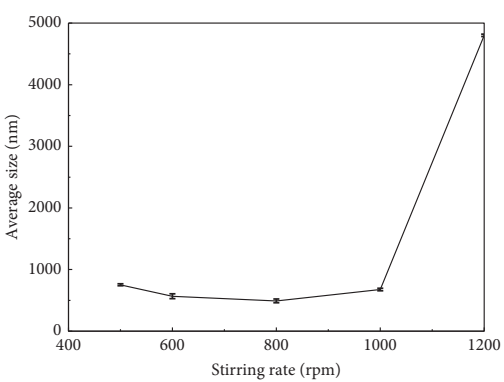

(c)

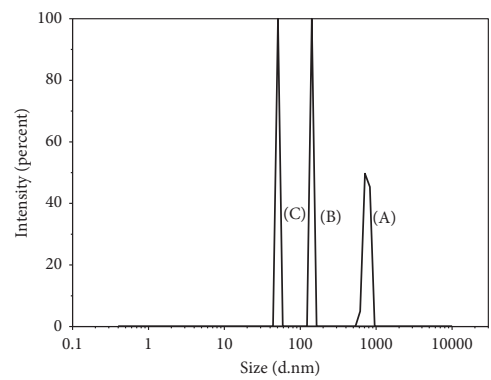

(f)

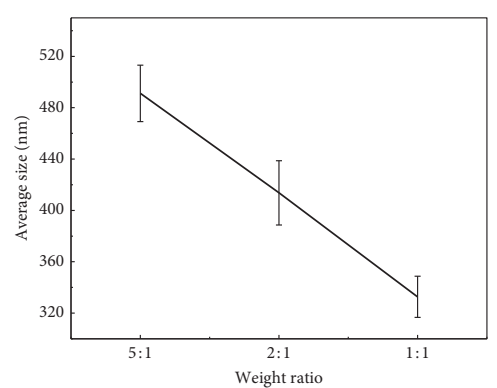

(g)

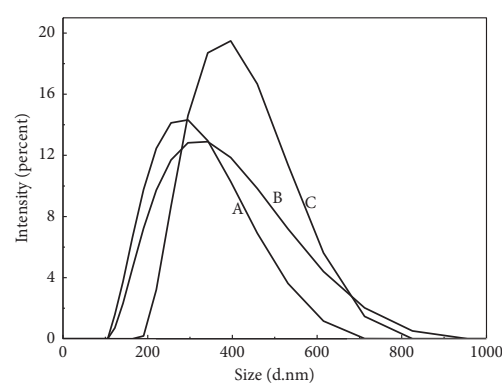

(h)

Figure 3: The average sizes (a) and size distributions (b) of the SA/chitosan particles loaded with CS at different flow rates of (A) $0.3 \mathrm{~mL} / \mathrm{h}$; (B) $0.6 \mathrm{~mL} / \mathrm{h}$; (C) $1.0 \mathrm{~mL} / \mathrm{h}$; (D) $1.2 \mathrm{~mL} / \mathrm{h}$. Average sizes (c) and size distributions (d) of the $\mathrm{SA} /$ chitosan particles loaded with CS at different stirring rates of (A) $600 \mathrm{rpm}$; (B) $800 \mathrm{rpm}$; (C) $1000 \mathrm{rpm}$. Average sizes (e) and size distributions (f) of the SA/chitosan particles loaded with CS at different applied voltages of (A) $13 \mathrm{kV}$; (B) $16 \mathrm{kV}$; (C) $18 \mathrm{kV}$. Average sizes (g) and size distributions (h) of the SA/chitosan particles loaded with CS at different weight ratios of SA to CS of (A) $5: 1$; (B) $2: 1$; (C) $1: 1$.

The average hydrodynamic size and distributions are shown in Figures 3(g) and 3(h). It can be found that the average size of SA/chitosan composite particles loaded with CS increased from $491.2 \pm 22.0 \mathrm{~nm}$ to $332.7 \pm 16.0 \mathrm{~nm}$, when the weight ratio of SA to CS decreased from $5: 1$ to $1: 1$, suggesting the average size of SA/chitosan composite particles loaded with CS decreased and size distribution moved towards the direction of small particle size with the increase of CS content in the composite particles. This may be due to the viscosity of the jet decreased with the decrease of the concentration, which made the jet easier to be torn into smaller particles at the same voltage and flow rate.

FTIR spectroscopy is a useful technique used to confirm the formation of an inclusion complex. The FTIR spectra of $\mathrm{SA} /$ chitosan micro/nanoparticles loaded with CS are shown in Figure 4. Peaks in the FTIR spectrum of the CS sample observed in Figure 4 are assigned according to the literature $[17,18]$. For example, axial $\mathrm{SO}_{3}$ stretching was found at

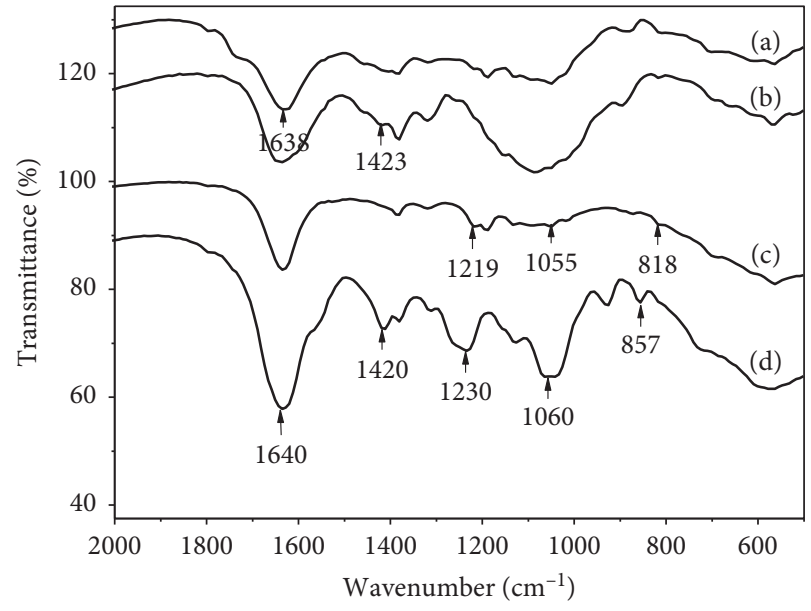

FIGURE 4: FTIR spectra of (a) chitosan; (b) SA; (c) CS; (d) SA/ chitosan nanoparticles loaded with CS. 
about $818 \mathrm{~cm}^{-1}$ and asymmetrical $\mathrm{S}=\mathrm{O}$ stretching of sulfate group bonds was observed at about 1055 and $1219 \mathrm{~cm}^{-1}$, respectively. The absorption peak at 1638 (amide I band) was found in native chitosan [19]. The spectrum of SA includes multiple characteristic peaks of carbohydrates. The broad peak at $3450 \mathrm{~cm}^{-1}$ corresponds to the stretching of hydroxyl groups $(\mathrm{O}-\mathrm{H})$, and the peak at about $1423 \mathrm{~cm}^{-1}$ was attributed to the carboxyl (-COO-) symmetrical stretching vibrations [20]. The FTIR spectrum of the SA/chitosan micro/nanoparticles loaded with CS sample (Figure 4(d)) was composed of the main bands found in the CA, chitosan, and CS samples, thus confirming the presence of CS in the $\mathrm{SA} /$ chitosan micro/nanoparticles. Moreover, the spectrum of the SA/chitosan micro/nanoparticles loaded with CS sample showed a systematic blue-shift except for the peak at about $1423 \mathrm{~cm}^{-1}$, which strongly suggests the interaction of the CS molecule with the SA/chitosan via hydrogen bond and electrostatic interactions.

\subsection{The Stability of SA/Chitosan Composite Particles Loaded} with CS. The Zeta potential of SA/chitosan composite particles loaded with CS in PBS $(\mathrm{pH}=6.8)$ buffer solution was $-34.5 \pm 3.5 \mathrm{mV}$, which means that the nanoparticles were not very stable in solution. This could be proved by the following two facts: one is that the particle sizes measured by SEM images were smaller than those measured by DLS; the second is confirmed by the fact that the average hydrodynamic size of the same sample increased and size distribution moved towards the direction of large particles with the extension of test time, as shown in Figure 5, indicating the SA/chitosan composite particles loaded with CS are unstable in water and suitable for oral medicine.

3.3. Swelling Study of Micro/Nanoparticles. Swelling ratios (SR) of CS@SA/chitosan nanocomposite hydrogels fabricated with different weight ratios of SA and CS under different $\mathrm{pH}$ values are shown in Figure 6. With the soaking time increasing from 0 to $8 \mathrm{~h}$, at $\mathrm{pH} 1.2$, the nanocomposite hydrogels (except weight ratios of SA and CS of $5: 1$ ) showed that negative SR with rapidly decreased (Figures 6(d) and 6(e)). In contrast, the SR of those with a weight ratio of SA and CS of 5:1 increased rapidly at the first $30 \mathrm{~min}$, then decreased slowly from $0.5 \mathrm{~h}$ to $3 \mathrm{~h}$, and then fluctuated up and down in a very low negative range (Figure 6(f)). At $\mathrm{pH}$ 6.8 , the SR of CS@SA/chitosan nanocomposite hydrogels first increased, then decreased rapidly, and then increased slowly, as shown in Figures 6(a)-6(c). These may be mainly related to the $\mathrm{pH}$ sensitivity of chitosan and the content of SA and CS. In acid buffer solutions, the SR of CS@SA/ chitosan nanocomposite hydrogels was determined by the swelling rate of SA and chitosan, the dissolution rate of chitosan, and the release rate of chondroitin sulfate, while in neutral buffer solutions, the SR of the hydrogels was determined by the swelling rate of SA and chitosan and the release rate of chondroitin sulfate. In addition, under the

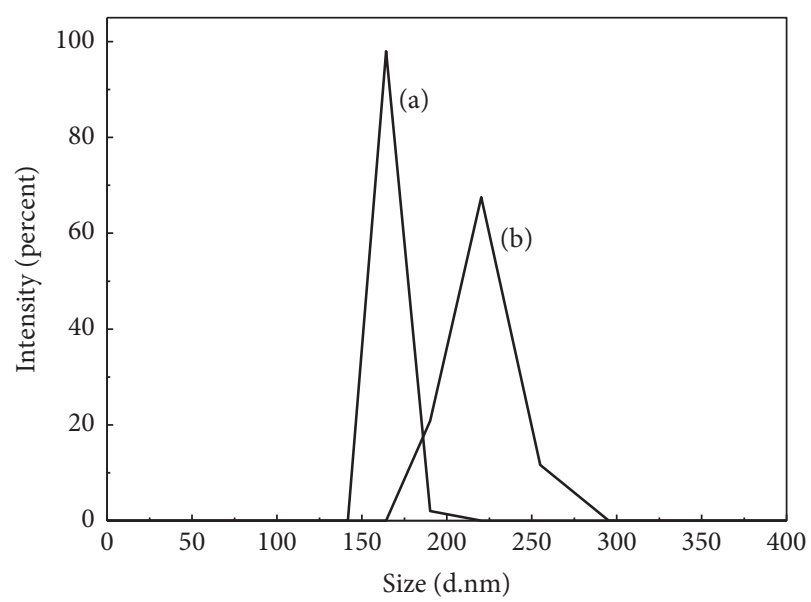

FIGURE 5: Size distributions of the SA/chitosan particles loaded with CS measured at different times: (a) after $1 \mathrm{~h}$; (b) after $3 \mathrm{~h}$ (the sample was prepared at the collected distance of $6 \mathrm{~cm}$, the stirring rate of $800 \mathrm{rpm}$, the flow rate of $0.6 \mathrm{~mL} / \mathrm{h}$, and applied voltage of $16 \mathrm{kV}$; the weight ratio of SA to CS was $5: 1$ ).

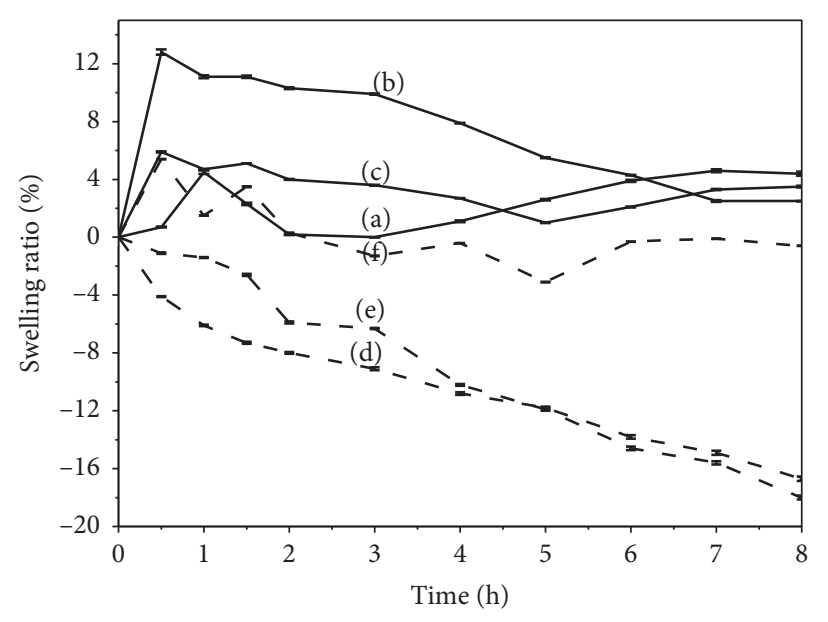

FIGURE 6: Swelling ratios in different pH values of CS@SA/chitosan nanocomposite hydrogels fabricated with different weight ratios of $\mathrm{SA}$ and CS at (a) $\mathrm{pH}=6.8,1: 1$; (b) $\mathrm{pH}=6.8,2: 1$; (c) $\mathrm{pH}=6.8,5: 1$; (d) $\mathrm{pH}=1.2,1: 1$; (e) $\mathrm{pH}=1.2,2: 1$; (f) $\mathrm{pH}=1.2,5: 1$.

same pH values, the SR of CS@SA/chitosan nanocomposite hydrogels was also affected by the weight ratio of SA and CS due to the variation of the content of $\mathrm{SA}$ and $\mathrm{CaCl}_{2}$ in each nanocomposite hydrogels, as shown in Figures 5(a)-5(c) and 6(d)-6(f). However, when the weight ratio of SA and CS was $5: 1$, the SR (165.0 $\pm 5.2 \%)$ of CS@SA/chitosan nanocomposite hydrogels in the buffer solution with $\mathrm{pH}$ value of 6.8 was lower than that $(205.0 \pm 10.5 \%)$ in the buffer solution with a $\mathrm{pH}$ value of 1.2 with soaking time extended to $144 \mathrm{~h}$. These results further showed that the outer membrane of alginate microspheres would slowly expand and form gel in acidic solution. Therefore, alginate microspheres can 
prevent the release of drugs in gastric acid, prolong the residence time in specific parts, and improve the utilization rate of drugs.

3.4. Release of CS from SA/Chitosan Composite Particles. The effects of different weight ratio of SA and CS and $\mathrm{pH}$ value of the buffer solutions on the cumulative release of the prepared micro/nanocomposite are presented in Figure 7.

3.4.1. The Effect of the Weight Ratio of SA and CS. From Figure $7(\mathrm{a})$, in the weight ratio of SA and CS of $2: 1$ in the buffer solutions with $\mathrm{pH} 7.4$, there was almost no release in the first $5 \mathrm{~h}$. Then, a rapid release period was observed with the cumulative release rate of $39.99 \pm 2.81 \%$ in $12 \mathrm{~h}$ and $40.27 \pm 2.76 \%$ in 24 hours. Moreover, a rapid release period with a cumulative release rate of $89.23 \pm 4.29 \%$ until $120 \mathrm{~h}$ occurred. For the weight ratio of SA and CS of $5: 1$ in the buffer solutions at a $\mathrm{pH}$ of 7.4, the release pattern is similar to that of the weight ratio of SA and CS of $2: 1$. However, the cumulative release rate of microcapsules was lower than that of the previous one. The cumulative release rate was $3.7 \pm 0.53 \%$ in the first $2 \mathrm{~h}, 31.9 \pm 1.56 \%$ in $12 \mathrm{~h}$, and $33.53 \pm 1.92 \%$ in $24 \mathrm{~h}$. Moreover, the cumulative release rate was only $77.66 \pm 4.62 \%$ in $120 \mathrm{~h}$, as shown in Figure $7(\mathrm{~b})$. These indicate that the release rate of SA/chitosan composite particles loaded with CS was affected by the weight ratio of SA and CS. The SA/chitosan composite particles loaded with CS with a high weight ratio of SA and CS had a lower release rate. These may be mainly related to the insolubility of chitosan coated on nanoparticles in neutral solvents, which prevents SA from absorbing solvents, swelling, and releasing the CS until the degradation of chitosan. After the degradation of the outer chitosan, the release resistance of CS is mainly related to the swelling of crosslinked SA. The higher the ratio of $\mathrm{SA}$ and $\mathrm{CS}$, the greater the resistance of $\mathrm{CS}$ release and the slower the release rate.

3.4.2. The Effect of $p H$. Under acidic conditions, the CS cumulative release rate from the SA/chitosan composite particles with a weight ratio of SA and CS of 2:1 was $14.46 \pm 1.33 \%$ in the first 2 hours, $17.92 \pm 1.04 \%$ in $12 \mathrm{~h}$, and $20.37 \pm 1.08 \%$ in $24 \mathrm{~h}$. Moreover, the release process was stable and slower than that of the SA/chitosan composite particles under neutral conditions. The cumulative release rate was only $51.42 \pm 3.12 \%$ in 120 hours, as shown in Figure $7(\mathrm{c})$. From the above analysis, it can be seen that under acidic conditions, the cumulative release rate of the composite particles had a rapid release phase in the first 2 hours and the release pattern and cumulative release rate of the composite particles are different under acidic conditions from under neutral conditions. These may be mainly related to the difference of solubility of chitosan and stretching of sodium alginate molecular chain in acidic and alkaline environments.

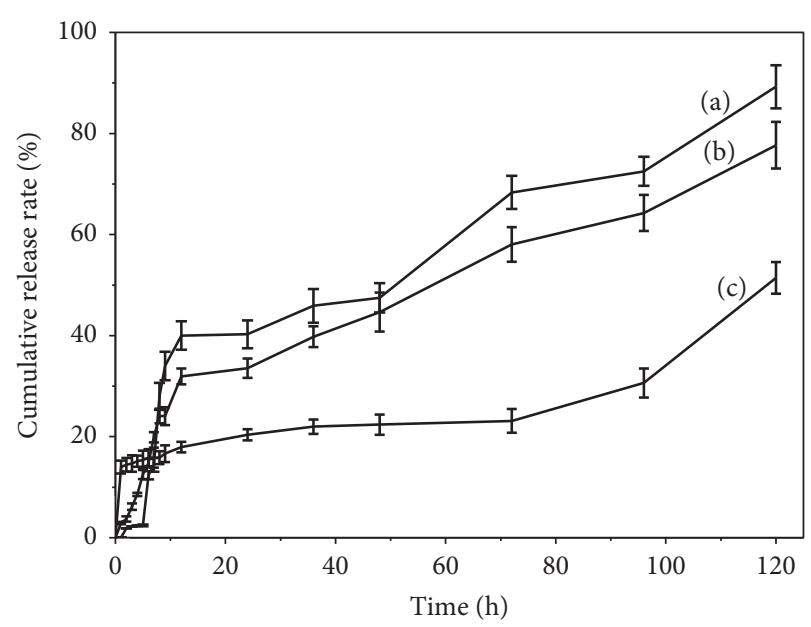

FIGURE 7: Relationship of the percent of cumulative release and time. (a) The weight ratio of SA and CS of $2: 1$ in the buffer solutions at a pH of 7.4; (b) the weight ratio of SA and CS of $5: 1$ in the buffer solutions at a $\mathrm{pH}$ of 7.4 ; (c) ghe weight ratio of SA and CS of $2: 1$ in the buffer solutions at a $\mathrm{pH}$ of 1.2 .

This is a desired release characteristic since the $\mathrm{pH}$ of tumor tissue and endosomes is acidic, whereas that of the blood stream and healthy tissues are neutral. Moreover, food passes through our bodies for 24 to $48 \mathrm{~h}$ from the time we eat it to the time we defecate. The food we eat takes about 2 to $5 \mathrm{~h}$ to pass through the stomach.

To further increase the CS cumulative release rate from the SA/chitosan composite particles with the weight ratio of SA to CS of $2: 1$ and $1: 1$, the weight ratio of SA to chitosan and calcium chloride was further optimized, respectively. Moreover, as shown in Figure 8, when the weight ratio of SA to chitosan and calcium chloride was adjusted to $1: 1$ and 3 : 17 , respectively, and the release of CS from the prepared CS/ SA/chitosan composite nanoparticles was characterized under the conditions simulating $\mathrm{pH}$, temperature, and time likely to be encountered during transit in the gastrointestinal tract. First, the dried nanoparticles were weighed, poured into a dialysis bag, and immersed in $50 \mathrm{~mL}$ of buffer solution at a $\mathrm{pH}$ of 1.2 for $2 \mathrm{~h}$ followed by immersing the dialysis bag into a buffered solution at a $\mathrm{pH}$ of 6.8 for $10 \mathrm{~h}$ to simulate gastric and intestinal media, respectively. From Figure 8(a), it was observed that the cumulative release rate of the composite particles with a weight ratio of SA to CS of $2: 1$ was only $15.30 \pm 0.11 \%$ in buffer solution at a $\mathrm{pH}$ of 1.2 for $2 \mathrm{~h}$ and then slowly released in buffer solution at a $\mathrm{pH}$ of 6.8 for $6 \mathrm{~h}$, the cumulative release rate was $22.37 \pm 0.19 \%$ and rapidly released during the time from $8 \mathrm{~h}$ to $12 \mathrm{~h}$, and the cumulative release rate reached $61.58 \pm 0.29 \%$ for $12 \mathrm{~h}$. These indicate that the most of the CS can pass through the stomach and be released in the intestinal tract. While the cumulative release rate of the composite particles with a weight ratio of SA to CS of $1: 1$ was $44.21 \pm 0.31 \%$ in buffer solution at a $\mathrm{pH}$ of 1.2 for $2 \mathrm{~h}$ and then slowly released in buffer solution at a $\mathrm{pH}$ of 6.8 for $10 \mathrm{~h}$; the cumulative release rate was $13.32 \pm 0.28 \%$; the cumulative release rate reached 


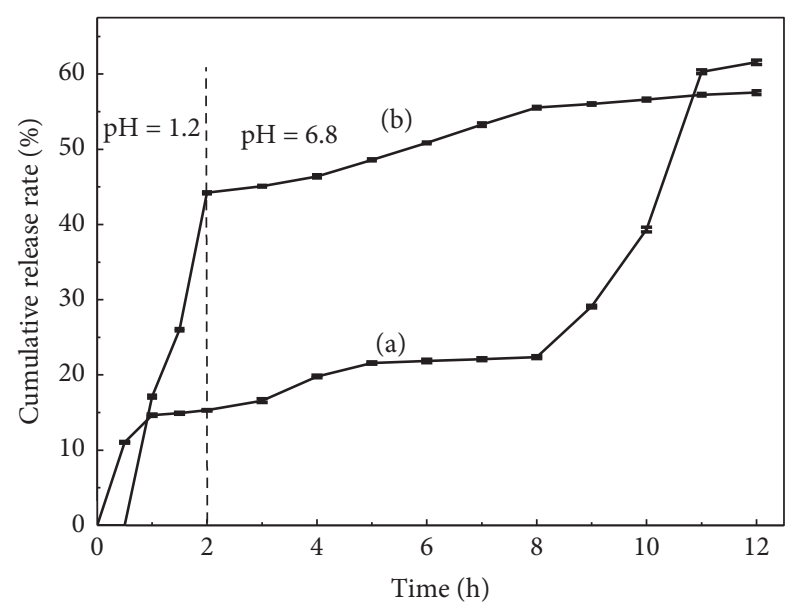

Figure 8: Cumulative release profiles of the CS/SA/chitosan composite nanoparticles prepared with a weight ratio of SA to CS of (a) $2: 1$ and (b) $1: 1$; SA to chitosan and SA to calcium chloride of 1 : 1 and $3: 17$, respectively, in simulated gastric $(\mathrm{pH}=1.2)$ and intestinal $(\mathrm{pH}=6.8)$ media at $37^{\circ} \mathrm{C}$.

$57.53 \pm 0.25 \%$ for $12 \mathrm{~h}$ (Figure $8(\mathrm{~b})$ ). It was likely that sodium alginate could not effectively coat chondroitin sulfate in the composite particles with a weight ratio of SA to CS of $1: 1$, resulting in a large amount of release of chondroitin sulfate after the dissolution of chitosan in acidic solution.

\section{Conclusions}

In this study, the feasibility of CS/SA/chitosan composite nanoparticles was successfully fabricated using electrostatic spraying technology. FTIR spectroscopy revealed that the CS, SA and chitosan were bound by hydrogen bond and electrostatic interactions. The size of SA/chitosan composite particles loaded with CS was strongly affected by process parameters. The size increased and size distribution moved towards the direction of large particle size with the decrease of applied voltage and increased with the increase of the flow rate and weight ratio of SA to CS; there is an optimal value for the effect of stirring rate on particle size and particle size distribution. The cumulative release rate of these particles was the weight ratio of SA to $\mathrm{CS}$ and $\mathrm{pH}$-dependent. The cumulative release rate of the $\mathrm{CS} / \mathrm{SA} /$ chitosan composite nanoparticles with a weight ratio of SA to CS of $2: 1$ was higher at $\mathrm{pH}$ of 6.8 than that at $\mathrm{pH}$ of 1.2. By optimizing the process parameters, the CS/SA/chitosan composite particles were prepared, in which most of CS could pass through the stomach and be released in the intestinal tract.

\section{Data Availability}

The data are available upon request to the corresponding author.

\section{Conflicts of Interest}

The authors declare that there are no conflicts of interest regarding the publication of this article.

\section{Acknowledgments}

This study was supported by the financial support of the National Science Foundation (Grant no. 51073072), the Open Project Program of Key Laboratory of Yarn Materials Forming and Composite Processing Technology of Zhejiang Province (No. MTC 2020-05), and the SRT Key Project of Jiaxing University (CD8517203299).

\section{References}

[1] N. Z. Nagy, Z. Varga, J. Mihály, G. Kasza, B. Ivan, and E. Kiss, "Highly efficient encapsulation of curcumin into and $\mathrm{pH}$ controlled drug release from poly( $\varepsilon$-caprolactone) nanoparticles stabilized with a novel amphiphilic hyperbranched polyglycerol," Express Polymer Letters, vol. 14, no. 1, pp. 90-101, 2020.

[2] Y. Li, J. Wang, M. G. Wientjes, and J. L.-S. Au, "Delivery of nanomedicines to extracellular and intracellular compartments of a solid tumor," Advanced Drug Delivery Reviews, vol. 64, no. 1, pp. 29-39, 2012.

[3] J. L.-S. Au, B. Z. Yeung, M. G. Wientjes, Z. Lu, and M. G. Wientjes, "Delivery of cancer therapeutics to extracellular and intracellular targets: determinants, barriers, challenges and opportunities," Advanced Drug Delivery Reviews, vol. 97, pp. 280-301, 2016.

[4] G. Pandey, N. Mittapelly, V. T. Banala, and P. R. Mishra, "Multifunctional glycoconjugate assisted nanocrystalline drug delivery for tumor targeting and permeabilization of lysosomal-mitochondrial membrane," ACS Applied Materials \& Interfaces, vol. 10, no. 20, pp. 16964-16976, 2018.

[5] X. Liu, Y. Liu, J. Hao et al., "In vivo anti-cancer mechanism of low-molecular-weight fucosylated chondroitin sulfate (LFCS) from sea cucumber cucumaria frondosa," Molecules, vol. 21, no. 5, pp. 625-632, 2016.

[6] K. M. Ilieva, A. Cheung, S. Mele et al., "Chondroitin sulfate proteoglycan 4 and its potential as an antibody immunotherapy target across different tumor types," Frontiers in Immunology, vol. 8, pp. 1911-1919, 2017.

[7] P. Liu, N. Chen, L. Yan et al., "Preparation, characterisation and in vitro and in vivo evaluation of CD44-targeted chondroitin sulphate-conjugated doxorubicin PLGA nanoparticles," Carbohydrate Polymers, vol. 213, pp. 17-26, 2019.

[8] P. du Souich, A. G. García, J. Vergés, and E. Montell, "Immunomodulatory and anti-inflammatory effects of chondroitin sulphate," Journal of Cellular and Molecular Medicine, vol. 13, no. 8A, pp. 1451-1463, 2009.

[9] M. Bishnoi, A. Jain, P. Hurkat, and S. K. Jain, "Chondroitin sulphate: a focus on osteoarthritis," Glycoconjugate Journal, vol. 33, no. 5, pp. 693-705, 2016.

[10] S. Ghosh, S. Ghosh, S. K. Jana, and N. Pramanik, "Biomedical application of doxorubicin coated hydroxyapatite-poly(lactide-co-glycolide) nanocomposite for controlling osteosarcoma therapeutics," Journal of Nanoscience and Nanotechnology, vol. 20, no. 7, pp. 3994-4004, 2020.

[11] H. B. Tan, F. Y. Wang, W. Ding et al., "Fabrication and evaluation of porous keratin/chitosan (KCS) scaffolds for effectively accelerating wound healing," Biomedical and Environmental Sciences: Biomedical and Environmental Sciences, vol. 28, no. 3, pp. 178-189, 2015.

[12] Z. C. Soe, B. K. Poudel, H. T. Nguyen et al., "Folate-targeted nanostructured chitosan/chondroitin sulfate complex carriers for enhanced delivery of bortezomib to colorectal cancer 
cells," Asian Journal of Pharmaceutical Sciences, vol. 14, no. 1, pp. $40-51,2019$.

[13] Y. Chen, V. J. Mohanraj, and J. E. Parkin, "Chitosan-dextran sulfate nanoparticles for delivery of an anti-angiogenesis peptide," Letters in Peptide Science, vol. 10, no. 5-6, pp. 621-629, 2003.

[14] K. C. Meltem and O. Murat, "Synthesis and controlled release of curcumin- $\beta$-cyclodextrin inclusion complex from nanocomposite poly( $\mathrm{N}$-isopropylacrylamide/sodium alginate) hydrogels," Journal of Applied Polymer Science, vol. 136, pp. 475541-475519, 2019.

[15] H. Wang, X. Gong, Y. Miao et al., "Preparation and characterization of multilayer films composed of chitosan, sodium alginate and carboxymethyl chitosan- $\mathrm{ZnO}$ nanoparticles," Food Chemistry, vol. 283, pp. 397-403, 2019.

[16] N. Mainreck, S. Brézillon, G. D. Sockalingum, F.-X. Maquart, M. Manfait, and Y. Wegrowski, "Rapid characterization of glycosaminoglycans using a combined approach by infrared and Raman microspectroscopies," Journal of Pharmaceutical Sciences, vol. 100, no. 2, pp. 441-450, 2011.

[17] J. Almodóvar, L. W. Place, J. Gogolski, and K. Erickson, "Layer-by-layer assembly of polysaccharide-based polyelectrolyte multilayers: a spectroscopic study of hydrophilicity, composition, and ion pairing," Biomacromolecules, vol. 12, no. 7, pp. 2755-2765, 2011.

[18] M. R. R. Luis, G. P. Leonardo, L. C. Natalia et al., "Biocompatible superparamagnetic carriers of chondroitin sulfate," Materials Research Express, vol. 6, pp. 066106-066115, 2019.

[19] N. Elsaid, T. L. Jackson, Z. Elsaid, A. Alqathama, and S. Somavarapu, "PLGA microparticles entrapping chitosanbased nanoparticles for the ocular delivery of ranibizumab," Molecular Pharmaceutics, vol. 13, no. 9, pp. 2923-2940, 2016.

[20] Q. Liu, Y. Jing, C. Han, and H. Zhang, "Encapsulation of curcumin in zein/caseinate/sodium alginate nanoparticles with improved physicochemical and controlled release properties," Food Hydrocolloids, vol. 93, pp. 432-442, 2019. 